\title{
Desenvolvimento de um Sistema de Armazenamento e Reconhecimento de Marcas de Proveniência em Livros Raros
}

\author{
Mateus A. Prado ${ }^{1}$, Alissa E. Vian ${ }^{2}$, Giancarlo Lucca ${ }^{1}$, \\ Marcia C. Rodrigues ${ }^{2}$, Eduardo N. Borges ${ }^{1}$ \\ ${ }^{1}$ Centro de Ciências Computacionais, Universidade Federal do Rio Grande \\ Av. Itália, Km. 8, CEP 96230-000, Rio Grande - RS \\ ${ }^{2}$ Instituto de Ciências Humanas e da Informação, Universidade Federal do Rio Grande \\ Av. Itália, Km. 8, CEP 96230-000, Rio Grande - RS \\ mp@furg.br, alissinhavian@hotmail.com, \\ \{giancarlo.lucca, marciabiblio, eduardoborges\}efurg.br
}

\begin{abstract}
This paper aims to present a system for storing and recognizing provenance marks in rare works composed of an online data repository, a mobile app and a web application server for image processing. A comparison is made between the SIFT and ORB characteristic detection algorithms in order to recognize a set of ex-libris marks.
\end{abstract}

Resumo. Este artigo tem por objetivo apresentar um sistema para armazenamento e reconhecimento de marcas de proveniência em obras raras composto por um repositório de dados online, um aplicativo mobile e um servidor de aplicação Web para processamento de imagens. Uma comparação entre os algoritmos de detecção de características SIFT e ORB é realizada com o objetivo de reconhecer um conjunto de marcas do tipo ex-libris.

\section{Introdução}

A preocupação com a segurança de acervos patrimoniais tem aumentado nos últimos anos, especialmente em decorrência do aumento dos índices de roubos e furtos em bibliotecas. A esse respeito, destaca-se a realização, em novembro de 2018, do XIII Encontro Nacional de Acervo Raro (ENAR), evento promovido pelo Plano Nacional de Recuperação de Obras Raras (PLANOR), da Fundação Biblioteca Nacional, que teve como tema "Políticas de segurança e salvaguarda de acervos raros e especiais". A questão da segurança dos acervos foi amplamente debatida e, a partir do evento, a Biblioteca Nacional passou a recomendar a todas as instituições que possuem acervos especiais e raros que façam uso de carimbos de identificação de propriedade em suas obras.

Marcas de proveniência definem a procedência de um livro raro. Esta proveniência refere-se ao registro de propriedade de um livro impresso ou manuscrito, o que pode incluir, também, a jornada que um livro impresso ou manuscrito realizou ao longo de sua vida, passando de um proprietário para outro [Overmier e Doak 1996].

A partir do exposto, torna-se evidente a necessidade do desenvolvimento de ferramentas que facilitem tanto o processo de registro destas marcas, especialmente por meio de imagens, quanto a sua posterior identificação e reconhecimento. Para esse fim foi 
desenvolvido o sistema apresentado neste trabalho, que foca na detecção e reconhecimento das marcas. Além disso, este trabalho contribui para dois dos grandes desafios de pesquisa em Sistemas de Informação no Brasil, uma vez que trata do gerenciamento de dados abertos ligados em ecossistemas inovadores e de um sistema complexo com componentes autônomos diversos e heterogêneos hospedados em diferentes plataformas computacionais.

\section{Estrutura do Sistema}

O sistema consiste de um repositório online, um aplicativo móvel e um servidor de aplicação Web. Utilizando o repositório, usuários de diferentes instituições podem fazer upload de novas marcas, consultar e colher metadados de marcas já disponíveis. A cada upload feito, o servidor de aplicação Web vai automaticamente reconhecer que uma nova marca foi registrada, processar a imagem e salvar seus descritores. Para não sobrecarregar os smartphones com processamento de imagens foi decidido utilizar esse servidor. Questões de interoperabilidade do repositório são resolvidas usando o software DSpace.

O aplicativo mobile é utilizado exclusivamente para captura das marcas de procedência. Para realizar o reconhecimento foi escolhido o algoritmo de detecção de características Oriented FAST and rotated BRIEF (ORB) [Rublee et al. 2011], que roda num servidor de aplicação Web. Após processar a foto registrada pelo usuário, o aplicativo retorna uma das seguintes mensagens: o link da marca de procedência no repositório ou, em caso de não reconhecida, um aviso que a marca precisa ser registrada. A Figura 1a apresenta a arquitetura do sistema de informações desenvolvido.

\section{Comparando ORB e SIFT}

Para garantir a eficácia do algoritmo escolhido para a detecção das marcas e atestar sua eficiência, foi realizada uma comparação com o bem conhecido algoritmo de detecção de características SIFT [Lowe 1999]. O conjunto de imagens utilizado no experimento é formado por 48 fotos diferentes de 18 marcas de procedência do tipo ex-libris capturadas da obra [Silva e Maciel 2014]. Para cada marca foram consideradas de 0 a 4 fotos distintas, com diferentes orientações, angulações, distâncias focais, luminosidades e smartphones. Essas variações foram intencionais devido a necessidade de testar os algoritmos de reconhecimento de padrões. A Figura $1 \mathrm{~b}$ apresenta exemplos de marcas iguais e uma distinta.
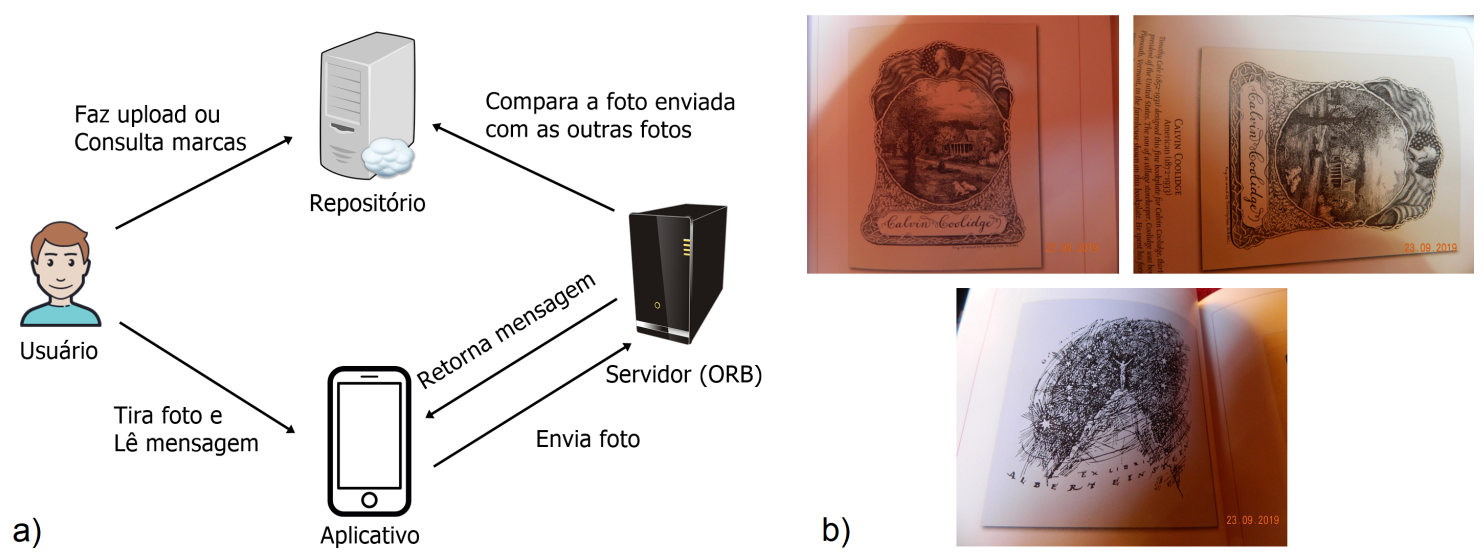

Figura 1. Arquitetura do sistema proposto (a) e exemplos de ex-libris (b). 
Foram realizados três experimentos para avaliar o desempenho dos algoritmos analisados. O primeiro foi referente ao tempo necessário para cada algoritmo detectar key points e computar os descritores das características extraídas de todas as imagens. Foram feitas 10 execuções de cada algoritmo e calculado o tempo médio. Key points são pixels relevantes representando cantos, bordas ou com determinadas propriedades. Já os descritores são conjuntos de características que descrevem os key points, extraídas com base na vizinhança destes pontos. Neste trabalho, os descritores são utilizados para avaliar a similaridade entre duas imagens, de forma a contornar variação de luminosidade, escala, rotação e angulação, tão comuns nas fotos feitas em dispositivos móveis.

O segundo experimento avalia o tempo necessário para calcular a similaridade de uma determinada imagem com todas as demais presentes no conjunto de dados. A similaridade é determinada pela média das distâncias entre descritores identificados pelo algoritmo como equivalentes para cada par de imagens. Assim como no primeiro experimento, foram realizadas 10 execuções de cada algoritmo e calculado o tempo médio.

No terceiro experimento foi calculada a média das precisões médias (Mean Average Precision - MAP) [Manning et al. 2008], com o objetivo de avaliar a qualidade da detecção de marcas duplicadas, ou seja, a capacidade dos algoritmos em reconhecer as marcas de procedência ex-libris em diferentes imagens. Nesta etapa foram removidas 2 fotos de marcas únicas, restando 46 imagens de 16 marcas distintas. Para cada imagem com repetição foi selecionada a foto com melhor qualidade e calculada a similaridade em relação às demais imagens. Após o cálculo, as imagens foram ordenadas em ordem crescente pela média da distância dos descritores detectados como equivalentes. Para cada uma das 16 marcas foi gerado um ranking de imagens similares, em que foi verificada a precisão para cada posição relevante, ou seja, onde existe uma marca equivalente. Por fim, é calculada a média das precisões médias (MAP) de cada ranking.

A Tabela 1 mostra os resultados de todos os experimentos realizados. Todos os experimentos foram codificados utilizando a linguagem de programação Python 3.7 e as implementações dos algoritmos da biblioteca OpenCV 2 em um único thread de um processador Intel Core i7-4510U 2.0 GHz. Observando os resultados percebe-se claramente o melhor desempenho do algoritmo ORB, escolhido para o reconhecimento de marcas neste projeto. SIFT é uma ordem de grandeza mais lento para detecção dos key points e até três ordens de grandeza mais lento no cálculo da similaridade entre os descritores. Quanto à qualidade do resultado, novamente ORB mostrou-se a melhor escolha, identificando quase que a totalidade das marcas de procedências ex-libris na primeira posição do ranking, ou seja, sem cometer falsos positivos.

Existem outros algoritmos que poderiam ter sido testados como, por exemplo, SURF e BRISK. A escolha do ORB e SIFT para esses testes foi devido à necessidade

Tabela 1. Resultado dos experimentos realizados.

\begin{tabular}{lrr}
\hline Algoritmo & ORB & SIFT \\
\hline Tempo médio para detecção de key points e & $3,88 \mathrm{~s}$ & $48,60 \mathrm{~s}$ \\
computação dos descritores & & \\
Tempo médio para cálculo de similaridade & $0,14 \mathrm{~s}$ & $242,97 \mathrm{~s}$ \\
Média das precisões médias (MAP) & $96,09 \%$ & $85,05 \%$ \\
\hline
\end{tabular}


de testar o maior contraste entre o algoritmo bem conhecido por sua precisão, o SIFT, e o algoritmo conhecido por ser o mais rápido de todos dessa classe, o ORB. Como queremos uma aplicação em tempo real, priorizamos a velocidade com que as tarefas são executadas, por isso mesmo que o ORB tivesse um desempenho similar ou um pouco pior ao SIFT na questão de precisão, optaríamos por ele.

\section{Considerações Finais}

O sistema de informações apresentado nesse trabalho busca desenvolver o Repositório Brasileiro de Marcas de Proveniência Bibliográficas, sendo o primeiro catálogo online voltado para o registro e difusão das marcas de proveniência presentes nos acervos bibliográficos pertencentes a pessoas físicas ou jurídicas residentes ou domiciliadas no Brasil [Carvalho et al. 2020]. Pretende-se que este Repositório seja de livre acesso a todos os interessados em integrar o projeto, e que os próprios integrantes colaborem para alimentar a base de dados, agregando informações sobre seus acervos de forma colaborativa, contribuindo para a criação de um catálogo coletivo nacional de marcas de proveniência bibliográficas. Foi implementado um repositório online para o registro das marcas, um aplicativo mobile para a identificação e reconhecimento destas marcas e um servidor de aplicação Web dedicado à identificação e ao reconhecimento. O sistema facilita o trabalho de bibliotecários e pesquisadores da área, possibilitando a verificação da existência de uma determinada marca em diferentes acervos e/ou instituições, bem como oferece uma alternativa de identificação e possível recuperação de exemplares perdidos ou roubados, por meio da aplicação do sistema desenvolvido para o reconhecimento das marcas.

Além de apresentar a arquitetura do sistema proposto, este artigo comparou o desempenho dos algoritmos de detecção de características ORB e SIFT para marcas de proveniência do tipo ex-libris. A partir da comparação feita, podemos concluir que a utilização do ORB com esse tipo de marca é preferível devido a velocidade superior com que o algoritmo executa as tarefas de encontrar pontos relevantes nas imagens, calcular seus descritores e calcular a similaridade entre imagens. O algoritmo ORB também apresentou maior confiabilidade no cálculo de similaridade das marcas, o que o torna a primeira opção na escolha de um algoritmo para a realização das tarefas exigidas.

\section{Referências}

Overmier, J. A. and Doak, E. M. (1996). Provenance records in rare book and special collections. Rare Books and Manuscripts Librarianship, 11(2):91-99.

Lowe, D. G. (1999). Object recognition from local scale-invariant features. In IEEE International Conference on Computer Vision, volume 2, pages 1150-1157.

Manning, C. D., Raghavan, P., and Schütze, H. (2008). Introduction to information retrieval. Cambridge university press.

Rublee, E., Rabaud, V., Konolige, K., and Bradski, G. (2011). Orb: An efficient alternative to sift or surf. In International Conference on Computer Vision, pages 2564-2571.

Silva, A. C. and Maciel, A. (2014). Livro dos ex-líbris. Rio de Janeiro: Academia Brasileira de Letras, São Paulo: Imprensa Oficial do Estado de São Paulo.

Carvalho, R. M., Esperon, V. A., and Heytor, D. T. (2020). Marcas de procedência: contribuições para o estudo do livro raro. Encontros Bibli: revista eletrônica de biblioteconomia e ciência da informação, 25:01-20. 